Reprod. Nutr. Dévelop. 1980, 20 (2), 523-537.

\title{
Le diagnostic prénatal dans les hémoglobinopathies humaines
}

\author{
par A. DUBART, M. GOOSSENS, Y. BEUZARD, N. MONPLAISIR, Y. DUMEZ *, \\ J. DUBUISSON*, R. HENRION*, J. ROSA (1) \\ Unifé de Recherches sur les Anémies \\ INSERM U.91. Hopital Henri-Mondor, 94010 Créteil, France \\ * Maternifé Port-Royal, Paris.
}

Summary. Prenatal diagnosis of human hemoglobinopathies.

About 1000 pregnancies risking sickle cell disease or thalassemia have now been diagnosed in 13 centers throughout the world. Up to now, the classical method for diagnosing these antenatal hemoglobinopathies has used fetal red blood cells obtained from the fetal blood in utero by fetoscopy or by aminocentesis when the former fechnique was impossible. The fetal blood sample, rich in reticulocytes, is incubated with a radioactive amino acid incorporated into the hemoglobin synthesized in the reticulocytes. Using biochemical techniques, the globin chains are separated by chromatography and the proportion of adult globin chains determined. The homozygous state in $\beta$ thalassemia is characterized by a complete depression of the $\beta^{A}$ chain synthesis or $a \beta A / \gamma$ ratio of globin chain synthesis below 0.02 . Fetuses heterozygous for $\beta$ thalassemia show a $\beta / \gamma$ ratio above 0.03 , while normal fetuses have a ratio higher than $6 \mathrm{p}$. 100. Sickle cell disease in the homozygous state is characterized by complete depression of $\beta^{A}$ chain synthesis with only $\beta^{S}$ chain synthesis present as the adult globin chain. When the fetal blood is contaminated by maternal blood, the adult red cells have to be discarded.

We have developed a new technique isoelectrically focusing the hemoglobins on acrylamide slab gels. This method is faster ans easier than the classical one and eliminates the use of radioactive material. It can be used in diagnosing prenatal sickle cell disease and thalassemias, but any maternal cells present must be completely eliminated.

Genetic engineering is a fascinating new approach which can already be applied in many cases of sickle cell disease diagnosis. The DNA of amniotic fluid cells is digested at specific sites by restriction enzymes, and the fragments are analysed according to size. The globin genes can be selectively characterized by specific and radioactive probes complementary to the $\beta$ globin genes. This method is not yet applicable to $\beta$ thalassemias, but has a great potential in the future.

\section{Introduction.}

Les techniques modernes de microanalyse des protéines ou d'étude des produits d'hydrolyse du DNA par des enzymes de restriction permettent actuellement de réaliser un diagnostic prénatal de la drépanocytose et des thalassémies. Ces affections sont des

(1) A qui toute correspondance doit être adressée. 
maladies hématologiques génétiquement déterminées, extrêmement graves pour lesquelles on ne dispose actuellement pas de thérapeutique satisfaisante. Les concepts et les technologies développées à ce propos servironi vraisemblablement de modèle au diagnostic prénatal de nombreuses autres affections humaines déterminées génétiquement ne pouvant actuellement bénéficier d'aucune thérapeutique curatrice.

La drépanocytose et les thalassémies sont des affections génétiquement déterminées, autosomales et récessives.

La drépanocytose est due à une mutation ponctuelle portant sur la chaîne $\beta$ de l'hémoglobine. II s'agit d'une substitution GluVal du 6e acide aminé à partir de l'extrémité $\mathrm{N}$ terminale. Contrairement aux autres hémoglobines normales ou anormales, l'Hb «S $»$ qui résulte de cette substitution a la propriété de former des polymères insolubles au cours de la déoxygénation. Ces polymères transforment les érythrocytes en hématies falciformes (fig. 1) dont la rigidité s'oppose à une rhéologie correcte dans les capillaires. Lorsqu'un phénomène de falciformation survient, le territoire irrigué devient ischémique et une nécrose apparaît. Les accidents ne surviennent que plusieurs mois après la naissance chez l'homozygote, car chez le nouveau-né l'existence dans les hématies à côté d'Hb $S\left(\alpha_{2}^{A} \beta_{2}^{S}\right)$ d'un pourcentage élevé d'Hb fœetale $(F)$ $\left(\alpha_{2}^{A} \gamma_{2}^{F}\right)$ prévient la formation des polymères.

- La présence d'Hb A $\left(\alpha_{2}^{\mathrm{A}} \beta_{2}^{\mathrm{A}}\right)$ dans les globules des hétérozygotes empêche également les accidents de survenir. Cette maladie est le triste apanage de l'ethnie 'noire et on la retrouve par conséquent partout où cette ethnie réside.
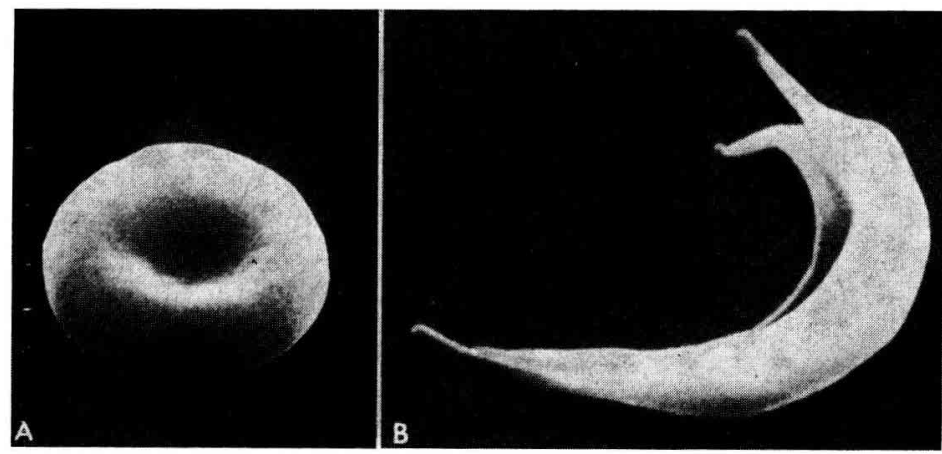

FIG. 1. - Scanning d'un globule d'un sujet drépanocytaire. A : en présence d'oxygène ; $B$ : en l'absence d'oxygène.

La défection des hétérozygotes est très facilement obtenue par électrophorèse de l'hémoglobine, l'Hb S étant chargée plus positivement que l'Hb A à pH alcalin. Malgré de grands efforts aucun traitement de la maladie n'a pu êire dégagé et les homozygotes décèdent plus ou moins rapidement après de nombreux accidents douloureux et invalidants. Les seules armes dont on dispose sont le conseil génétique, généralement très difficile à mettre en œuvre dans les populations touchées, ou le diagnostic prénatal.

Les thalassémies ne sont pas caractérisées par la synthèse d'une hémoglobine anormale. Ce sont des anémies hémolytiques dues à une assez grande variété d'accidents génétiques différents et qui n'ont d'ailleurs pas encore été totalement identifiés, 
bien que leur analyse soit très activement poussée grâce à la technologie moderne d'analyse des gènes.

Quelle que soit la lésion au niveau du DNA, les thalassémies sont caractérisées par une synthèse insuffisante ou nulle d'un des 2 principaux types de chaînes constitutives de l'hémoglobine : la chaîne $\beta$ et la chaîne $\alpha$. On dit que l'on est en présence respectivement d'une $\beta$ ou d'une $\alpha$ thalassémie. Le déséquilibre entre les chaînes de type $\alpha$ et de type $\beta$ entraîne d'abord une concentration insuffisante d'Hb, c'est-à-dire une anémie mais surtout un raccourcissement considérable de la durée de vie des hémalies, les chaînes synthéisées normalement ne trouvant pas un nombre suffisant de chaînes de l'autre type. Ne pouvant s'apparier asymétriquement, elles précipitent sur la membrane qui esi détériorée, provoquant le raccourcissement de la vie de l'érythroblaste et une érythropoièse qui bien que très fortement accrue reste inefficace.

Le diagnostic anténatal, dans l'état actuel des possibilités thérapeutiques, constituait une orientation prioritaire. L€s problèmes français dans ce domaine sont divers. Dans les départements antillais le nombre annuel de grossesses à risque drépanocytaire est de l'ordre de 200. La Réunion a vraisemblablement des problèmes de même ordre de grandeur. On sait que l'incidence des thalassémies dans la population Corse est de l'ordre de 3 p. 100 (20). De nombreux couples à risques d'origine méditerranéenne vivent par ailleurs en France. Nous sommes enfin sollicités par les pays africains francophones, sièges d'une très forte incidence de drépanocytose. Ces différentes considérations, la fréquence avec laquelle nous éitions interrogés, nous ont amenés voici deux ans, à mettre sur pied le diagnoslic prénatal des hémoglobinopathies.

La détection in utero des homozygotes $\beta$ thalassémiques ou drépanocytaires a d'abord requis l'obtention d'hématies fœtales car les gènes de l'hémoglobine ne s'expriment pas dans les autres cellules de l'organisme. Dès 1973, les travaux de Kan et Valenti $(13,22)$, puis de Hobbins et Mahoney $(10,11)$, laissaient envisager la possibilité de prélever du sang fœetal pendant la grossesse. Depuis 1974, plusieurs centaines de détections anténatales, la plupart de thalassémie, ont été pratiquées dans cinq ou six centres répartis dans le monde ; une quarantaine de ces diagnostics ont à ce jour fait l'objet d'une publication $(1,15)$. De très grands progrès ont été récemment réalisés dans diverses directions :

- qualité du repérage du fœelus et de ses annexes grâce à l'amélioration des techniques d'échographie et du matériel de fœtoscopie ;

- utilisation d'analyseur de volume des particules permettant de connaître en « per opératoire » la composition exacie du prélèvement (appréciation du degré de contamination du prélèvement par les globules maternels) ;

- l'élimination des globules maternels dans le prélèvement est devenue si efficace (2) que la transfusion préalable des mères peut maintenant être évitée ;

- l'analyse du prélèvement est en passe d'être considérablement simplifiée (la synthèse in vitro et la séparation chromatographique des chaînes vont devenir inutiles si la technique de l'isofocalisation prénatale (7) confirme ses promesses).

Enfin de très grands espoirs sont nés des résultats des recherches entreprises quant aux possibilités d'effectuer le diagnostic prénatal sur les fibroblastes du liquide amniotique. La culture des fibroblastes suivie d'un dosage de gènes (14) utilisée par Kan pour la détection des homozygoles $\alpha$ thal. a ouvert la voie dans les recherches de ce type. 
L'utilisation très récente d'enzymes de restriction suivie de la technique du « blotting » laisse entrevoir des perspectives entièrement nouvelles en matière de diagnostic prénatal pouvant peut-être permettre une très large diffusion de celui-ci géographiquement (il ne nécessiterait plus localement que l'infrastructure nécessaire à une amniocentèse), et peut-être même conceptuellement, cette technique ouvrant peut-être la voie vers un très grand nombre d'autres possibilités de diagnostics prénataux.

\section{I. - Le diagnostic prénatal « classique ».}

\section{A. - Aspects hématologiques ef biochimiques.}

La possibilité de détecter in utero des affections qui, comme la drépanocytose ou la $\beta$ thalassémie, sont dues à une anomalie de la chaîne $\beta$ de l'hémoglobine était fonction de deux questions préalables :

1) Quelle est la quantité exacte de chaîne $\beta$ produite par le foetus au cours du premier semestre?

2) Est-ce que ces hémoglobinopathies s'expriment déjà chez le fœetus à cette période, permettant leur diagnostic in ufero?

La synthèse de l'Hb A $\left(\alpha_{2}^{\mathrm{A}} \beta_{2}^{\mathrm{A}}\right)$ chez le fotus a été largement documentée au cours des quinze dernières années. L'aspect des hémoglobines présentes dans les hématies de l'embryon, puis du fœetus, se modifie avec l'âge gestationnel (fig. 2). L'hémoglobine fœetale $\left(\alpha_{2}^{\mathrm{A}} \gamma_{2}^{\mathrm{F}}\right)$ est associée dans les toutes premières semaines à différentes hémoglobines embryonnaires ( $\mathrm{Hb}$ Portland, Gower 1 et 2 ) dont la synthèse cesse vers la 6e semaine. La production de l'hémoglobine adulte $(\mathrm{Hb} \mathrm{A})$ débute à cette époque ef augmente régulièrement pour conslituer $30 \mathrm{p}$. 100 de l'hémoglobine présente dans le sang du nouveau-né. Entre la $16^{\circ}$ et la $20^{\circ}$ semaine (époque où l'on pratique le diagnoslic) un dixième de l'hémoglobine synthétisée est constitué par l'hémoglobine $A$, le reste étant de l'Hb F. Les quantités d'hémoglobine que l'on obtient en prélevani in utero étant infimes la méthode a donc consisté en une amplification préalable par « synthèse

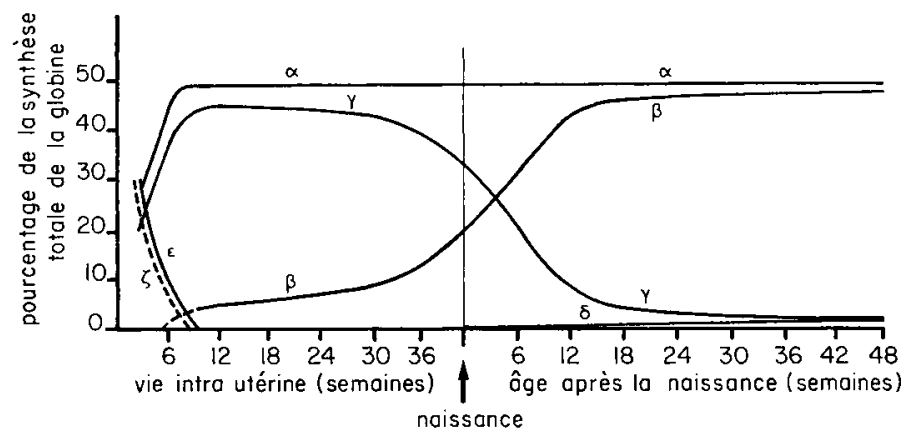

FIG. 2. - Synthèse des choines de globine pendant le développement humain.

La composition des différentes hémoglobines est la suivante :

a) Hbs embryonnaires Gower $1\left(\zeta_{2} \varepsilon_{2}\right)$, Gower $2\left(\alpha_{2} \varepsilon_{2}\right)$ et Portland $\left(\zeta_{2} \gamma_{2}\right)$. b) Hb fœtale $\left(\alpha_{2} \gamma_{2}\right)$. c) Hbs adultes $A\left(\alpha_{2} \beta_{2}\right)$ et $A_{2}\left(\alpha_{2} \zeta_{2}\right)$. 
in vitro ». Les hématies fœtales sont incubées en présence d'un précurseur radioactif. II y $a$, pendant l'incubalion, synthèse des diverses hémoglobines $(A, F, S$, etc...) par les réticulocytes dont le nombre est élevé (20-25 p. 100) chez le fœtus de 20 semaines. Les chaînes constitutives sont séparées par chromatographie sur résine échangeuse d'ions, et l'examen du profil d'élution de la radioactivité incorporée dans la globine $\gamma, \beta$ et $\alpha$ (fig. 3) permet de déceler les diverses anomalies de structure ou de synthèse de la

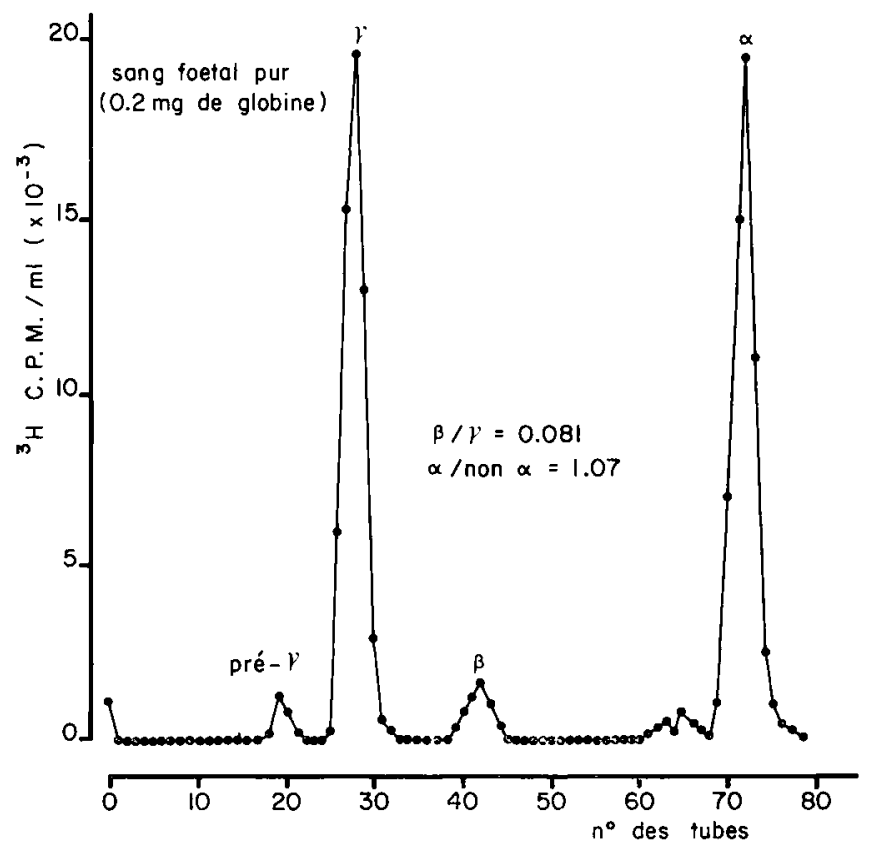

FIG. 3. - Radiochromatographie sur $C M$ cellulose-urée $8 M$ des chaines de globine d'un fœetus de 20 semaines. Le sang fœtal a été obtenu par fœtoscopie et l'hémoglobine marquée pendant l'incubation des cellules avec de la leucine tritiée.

FIG. 4. - Rapports $\beta / \gamma$ déterminés pour des faetus normaux et des foetus $\grave{a}$ risque pour lo $\beta$ thalassémie majeure. Le sang fœital a été obtenu par placentocentèse ou foetoscopie à la maternité Port-Royal à Paris. Certains prélèvements ont été effectués à Londres (University College Hospital et King's College Hospital).

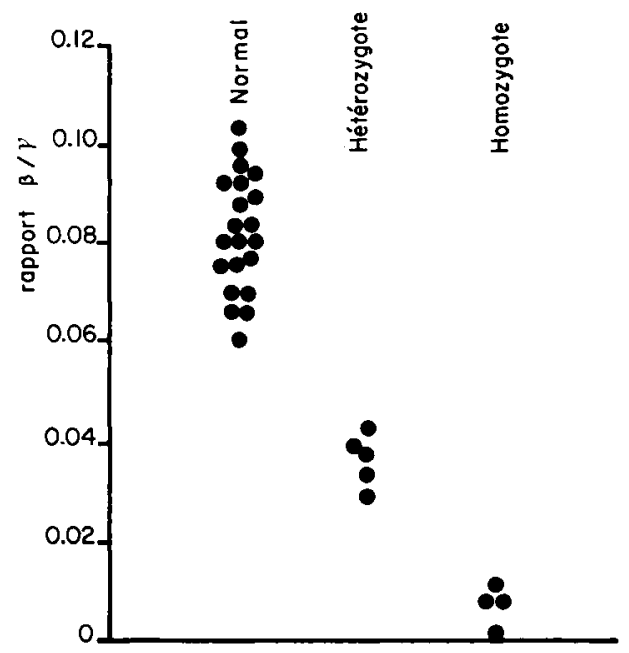


chaîne $\beta$. Plusieurs éłudes ont confirmé que les gènes $\beta^{S}$ et $\beta^{\text {thal. }} s^{\prime}$ expriment au cours du premier trimestre $(5,16)$ et qu'il serait possible de mettre en évidence toute anomalie moléculaire de l'hémoglobine transmise par les parents au fœetus dès la 8 e semaine de la vie gestationnelle.

La proporlion d'Hb A synthétisée peut être déterminée par le calcul du rapport $\beta / \gamma$ (rapport de la radioactivité totale incorporée dans les chaînes $\beta$ à la radioactivité totale $\gamma$ ). Il existe une bonne corrélation entre ce quotient et l'âge gestationnel (6). Entre la $16^{\mathrm{e}}$ et la $20^{\mathrm{e}}$ semaine, la valeur normale de ce rapport, telle qu'elle a été déterminée à partir de fœtus indemnes de toute affection hématologique, est comprise pour notre laboratoire, entre 0,06 et 0,10 (fig. 4).

Les lésions moléculaires sont de plusieurs sortes ef leur diagnostic est de difficulté variable :

a) Drépanocytose. - Le diagnostic prénatal de la drépanocylose homozygoie est simple; on constate l'absence totale de chaîne $\beta^{A}$ et la présence de chaîne $\beta^{S}$. En cas d'hétérozygote $\mathrm{A} / \mathrm{S}$ ou $(\mathrm{S} / \mathrm{C})$, à côté d'une chaîne $\beta^{\mathrm{S}}$, on détecte la présence d'une chaîne $\beta^{A}$ (ou $\beta$ C) (fig. 5).

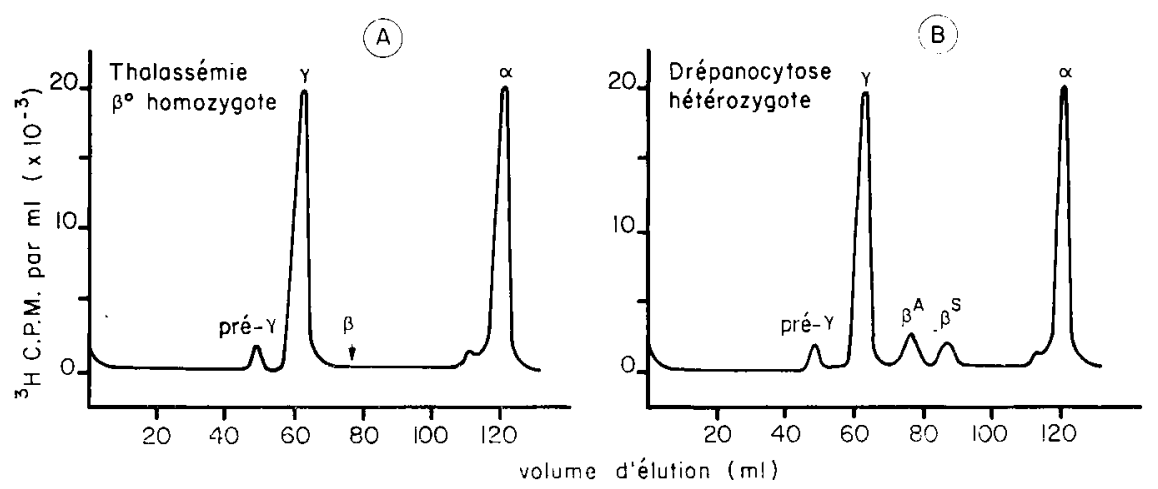

FIG. 5. - Séparation des chaînes de globine de deux fœtus de 20 semaines.

a) Homozygote $\beta^{\circ}$ thal. démontré par l'absence de synthèse de chaîne $\beta^{A}$. b) Hétérozygote $A / S$ : il y a synthèse de deux types de chaînes $\beta^{A}$ et $\beta^{S}$.

b) Les $\beta$ thalassémies. - La forme habituelle de $\beta$ thalassémie (associée à une augmentation du taux de l'Hb $A_{2}$ chez l'hétérozygote) peut être classée en deux types selon la présence ou l'absence de synthèse de chaîne $\beta$ à l'état homozygote. Cette synthèse, très diminuée dans les $\beta^{+}$thalassémies, est absente chez les patients atieints de $\beta^{0}$ thalassémie. Le diagnostic prénatal de $\beta^{0}$ thalassémie ne présente aucune difficulté car l'absence totale de synthèse $\beta$ permet d'affirmer l'état homozygote (fig. 5a). La possibilité de détecter in utero les formes $\beta^{+}$était difficile à prévoir car l'on manquait de données quantitatives sur l'expression du gène $\beta^{+ \text {thal }}$ au cours du 2 e trimestre de la grossesse. Les fotus homozygotes pour ce type d'affection produisent de l'Hb A à un taux faible, inférieur à celui des hétérozygotes, dont il faut les différentier. II ressort de l'expérience des centres qui ont maintenant des années de recul dans la pratique du diagnostic anténatal que le taux de production de l'Hb A est suffisamment faible, chez 
le fotus atleint de thalassémie $\beta^{+}$homozygote, pour qu'il n'y aił pas d'ambiguité dans la distinction entre homozygotes et hétérozygotes (fig. 4).

B. - Problèmes posés par le prélèvement du sang fœal.

1) Méthodes de prélèvement.

Les actes permettant ce prélèvement doivent être le moins traumatiques possible pour la mère et l'enfant car les résultats du diagnostic conduisent 3 fois sur 4 à la poursuite de la grossesse. Deux approches méthodologiques ont été conjointement développées : le prélèvement sous contrôle fœetoscopique et la ponction aveugle du placenta ou placentocentèse.

a) La date de prélèvement se situe entre la $16^{\mathrm{e}}$ et la $22^{\mathrm{e}}$ semaine. Le placenta est d'abord localisé dans la cavité utérine par échographie ultrasonore (fig. 6) et son mode d'insertion - antérieur ou postérieur - est soigneusement précisé.

b) La ponction du placenta a été la première technique utilisée. Elle est encore employée lorsque le placenta recouvre toute la face antérieure et ne laisse aucune «lucarne». L'opérateur procède comme pour une amniocentèse précoce, mais il arrête la pointe de son aiguille dans l'épaisseur du placenta et effectue une série d'aspirations en changeant à chaque fois la haułeur et/ou la position de l'aiguille. Chacune ramène un très faible volume de sang placentaire $(0,05 \mathrm{ml}$ à $0,1 \mathrm{ml})$.

c) La fœtoscopie a l'avantage de permettre le contrôle visuel du prélèvement. Elle est utilisée lorsque le fœłoscope peut être introduit sans léser le placenta. La moindre goutte de sang rend toute vision impossible. Le trocard est introduit après anesthésie locale ou générale dans la cavité utérine par voie transabdominale. C'est un endoamnioscope de faible calibre équipé d'une aiguille permettant de ponctionner une

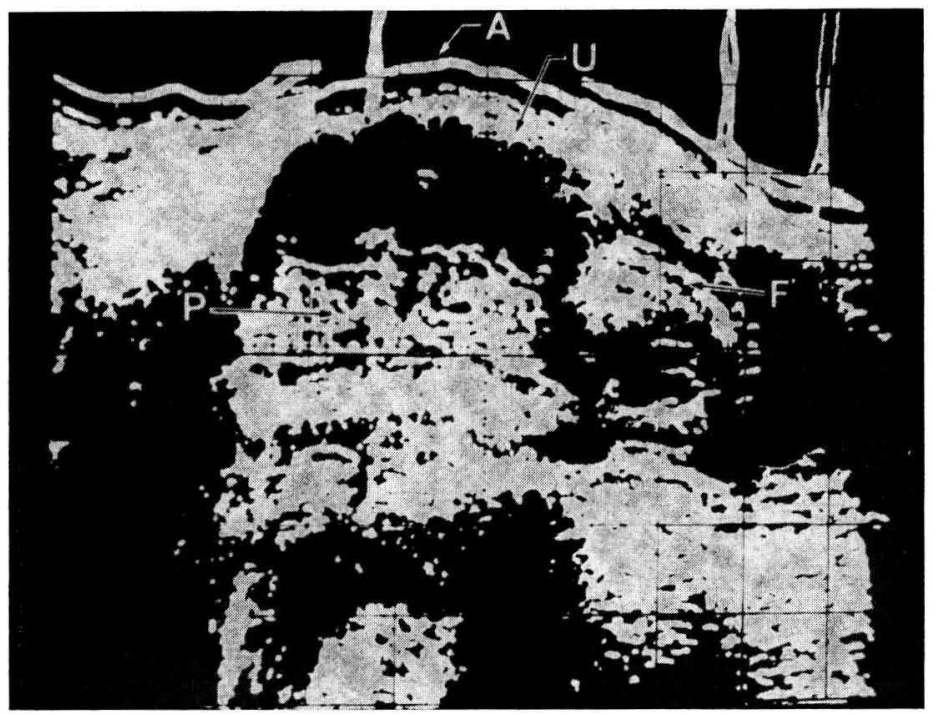

FIG. 6. - Echographie d̀ la 20e semaine de gestation.

$A$ : paroi abdominale ; $U$ : paroi utérine ; $P$ : placenta ; $F$ : føetus. 
veine fœtale de la surface de la plaque choriale et d'aspirer du sang très riche en hématies fœtales ou du sang fœetal pur, parfois dilué par du liquide amniotique. La rigidité de l'ensemble du système rend l'utilisation du fœtoscope difficile quand le placenta est inséré sur la face antérieure de l'utérus.

2) La purification du sang placentaire.

Quand l'échantillon est contaminé par le sang maternel, il ne peut être traité sans purification préalable, car les réticulocytes du sang maternel incorporent aussi le précurseur isotopique et produisent donc des chaînes de type adulte, radioactives. Ils peuvent par exemple, lorsqu'ils sont trop abondants, être à l'origine d'un rapport de synthèse $\beta / \gamma$ supérieur à celui qui serait obtenu à partir de sang fœetal pur, et qu'il y a lieu de corriger. Cette correction est nécessaire toutes les fois où le pourcentage des cellules fœtales du prélèvement est inférieur à 30 p. 100.

La teneur en cellules foilales de chaque prélèvement doit être évaluée immédiatement en salle d'opération en utilisant un analyseur de particules (Channelyser Coultronics). Le volume globulaire moyen des hématies fœtales est largement supérieur à celui des globules rouges de la mère et l'appareil affiche en quelques secondes, sur un oscilloscope, la distribution des volumes cellulaires (fig. 7). La coloration des hématies fœilales sur lame (Test de Kleihauer) effectuée ultérieurement à titre de contrôle ne permet cette estimation qu'en 15 à 20 min et consiste à incuber l'étalement à pH acide

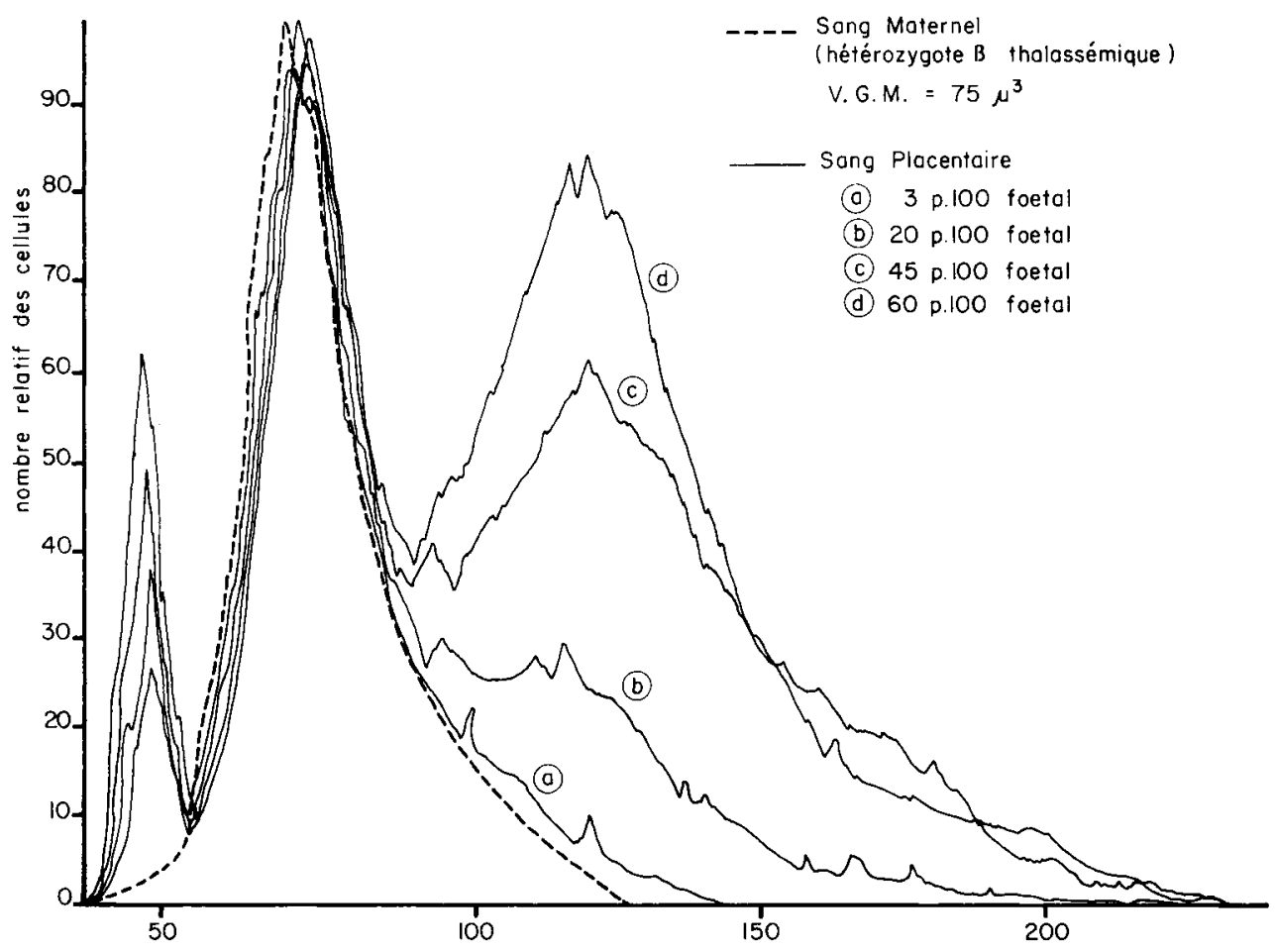

FIG. 7. - Courbes de distribution des volumes globulaires obtenues avec l'analyseur de volume de particules lors d'un prélèvement in utero par aspiration placentaire. 
puis à colorer : l'Hb A est éluée, seule persiste l'Hb F qui rend visible les globules fœtaux. Si les prélèvements obtenus contiennent un pourcentage élevé d'hématies maternelles, différentes méthodes permettent de corriger cefte contamination :

- L'utilisation d'anti-sérums anti-i ou anti-I (Les hématies foefales portent à leur surface l'antigène membranaire $i$, alors que les globules rouges adultes ont l'antigène l) permet d'agglutiner de façon spécifique respectivement les globules rouges fœtaux ou adultes $(8,9,12)$. Les agglutinats sont séparés des cellules libres et l'on peut enrichir ainsi en cellules fotales un échantillon placentaire qui contient un grand nombre d'hématies maternelles.

- Il est possible de déterminer le rapport de synthèse $\beta / \gamma$ du fotus en diluant le sang prélevé par différents volumes de sang maternel avant l'incubation des cellules (6). Le rapport $\beta / \gamma$ étant fonction de l'inverse du pourcentage en cellules fœtales de chaque dilution, celui du sang fœtal pur peư être ainsi graphiquement extrapolé.

- L'hémolyse sélective des globules rouges maternels par la méthode de ØrskovJacobs-Stewart telle qu'elle a été adaptée (2) (4) permet un enrichissement total. Cette méthode tire profit des concentrations différentes en anhydrase carbonique des cellules adultes et fœtales. L'hémolyse des globules maternels est très rapide en présence de concentrations adéquates d'ions bicarbonates, de chlorure d'ammonium et d'acétozolamide (un inhibiteur de l'anhydrase carbonique) alors que les cellules fœtales, dans les mêmes conditions, sont plus lentement hémolysées.

C. - Résultats (7).

Le tableau 1 représente à titre d'exemple les enrichissements en cellules fœtales

TABLEAU 1

Enrichissement en globules rouges fœiaux par lyse sélective des cellules maternelles présentes dans les prélèvements

\begin{tabular}{|c|c|c|c|c|}
\hline Sujets & $\begin{array}{l}\text { Grossesse } \\
\text { à risque }\end{array}$ & $\begin{array}{l}\text { Génotype } \\
\text { du fœetus }\end{array}$ & $\begin{array}{l}\text { Proportion initiale de } \\
\text { cellules fœetales } \\
\text { (p. 100) }\end{array}$ & $\begin{array}{l}\text { Proportion finale de } \\
\text { cellules foetales } \\
\text { (p. 100) }\end{array}$ \\
\hline MO. & $\beta$ thal. & $A / \beta$ thal. & 3 & 100 \\
\hline LOS. & S & $\mathrm{A} / \mathrm{S}$ & 2 & 100 \\
\hline LOM. & $\beta$ thal. & $A / A$ ou $A / \beta$ thal. & 30 & 100 \\
\hline PA. & $S$ & $\mathrm{~A} / \mathrm{A}$ & 85 & 100 \\
\hline DE. & $\mathrm{S}$ & $\mathrm{A} / \mathrm{S}$ & 90 & 99,7 \\
\hline FR. * & Hémophilie & $\mathrm{A} / \mathrm{A}$ & * $\begin{array}{r}5 \\
10 \\
20 \\
40\end{array}$ & $\begin{array}{c}100 \\
100 \\
99,5 \\
99\end{array}$ \\
\hline ON. & s & $\mathrm{A} / \mathrm{S}$ & 10 & 100 \\
\hline SE. & $S$ & $\mathrm{~A} / \mathrm{S}$ & 5 & 99,3 \\
\hline MA. & Hémophilie & $\mathrm{A} / \mathrm{A}$ & 5 & 98,5 \\
\hline
\end{tabular}

S : Drépanocytose ; * Mélange artificiel. 
obtenus avec 4 mélanges artificiels, cellules maternelles/fœtales (cas FR.) et avec 8 prélèvements où la contamination maternelle était importante. On voit qu'à 2 exceptions près (SE. et MA.) la contamination maternelle a été réduite à $0,5 p$. 100 ou moins, ce qui rend le diagnostic très aisé. La prolongation du temps de lyse doit permettre d'obtenir ce résultat même quand le pourcentage de départ des globules fœtaux est très faible.

Pour ce qui est du diagnostic prénatal proprement dit, nos propres résultats figurent sur le tableau 2. Les valeurs normales ont été obtenues à partir de prélèvements effectués après l'accord des parents, au cours de 10 fotoscopies entreprises pour des risques génétiques n'impliquant pas d'anomalies du système hémoglobine (myopathies, hémophilies). Cette table donne les résultats de synthèse obtenus au cours des 15 premiers cas de diagnostic prénataux effectués par notre équipe. Sur les 8 prélèvements à risque drépanocytaire, nous avons trouvé 4 fœitus $A / A$ et 4 fœtus $A / S$ et laissé les grossesses se poursuivre. Quatre diagnostics ont déjà été confirmés à la naissance. Sur les 7 prélèvements effectués au cours de grossesses à risque thalassémique, nous avons trouvé 2 homozygotes pour qui l'interruption de grossesse a eu lieu, et où le diagnostic a été confirmé sur le sang du fœetus. Deux prélèvements ont été interprétés comme provenant de fœtus hétérozygotes, 3 de fœtus normaux.

TABLEAU 2

Rapport des radioactivités incorporées dans des chaines de globines d'échantillons de sang fœetal

\begin{tabular}{|c|c|c|c|}
\hline Sujets & $\begin{array}{l}\text { Grossesse } \\
\text { à risque }\end{array}$ & $\begin{array}{c}\text { Rapport } \\
\beta_{A} / \gamma\end{array}$ & $\begin{array}{c}\text { Rapport } \\
\beta_{\mathrm{S}} / \gamma\end{array}$ \\
\hline OM. $\ldots \ldots \ldots \ldots$ & Drépanocytaire & 0,082 & \\
\hline EN. $\ldots \ldots \ldots \ldots \ldots$ & 一 & $0, \overline{084}$ & \\
\hline TA. $\ldots \ldots \ldots \ldots \cdots$ & - & 0,042 & \\
\hline PA. . . . . . . . . & - & 0,063 & \\
\hline LOS. $\ldots \ldots \ldots \ldots$ & - & 0,062 & 0,026 \\
\hline DE. $\ldots \ldots \ldots \ldots \ldots$ & - & 0,047 & 0,020 \\
\hline ON. $\ldots \ldots \ldots \cdots \cdots$ & - & 0,047 & 0,021 \\
\hline SE. $\ldots \ldots \ldots \ldots \ldots$ & - & 0,044 & 0,025 \\
\hline FE. $\ldots \ldots \ldots \ldots \ldots$ & Thalassémique & 0,070 & \\
\hline LOM. . . . . . . . . & - & 0,061 & \\
\hline TS. $\ldots \ldots \ldots \ldots \ldots$ & - & 0,057 & \\
\hline PI. $\ldots \ldots \ldots \ldots \ldots$ & - & 0,037 & \\
\hline PO. $\ldots \ldots \ldots \ldots \ldots$ & - & 0,034 & \\
\hline ZE. $\cdots \ldots \ldots \ldots \ldots$ & - & 0,012 & \\
\hline BO $\ldots \ldots \ldots \ldots \ldots$ & - & 0,016 & \\
\hline 10 échantillons $\ldots \ldots$ & Contrôles & $\begin{array}{l}\text { Moyenne } \\
0,085\end{array}$ & \\
\hline
\end{tabular}


Cinq des 8 diagnostics pour risque drépanocytaire ont été pratiqués chez des mères guadeloupéennes, 2 étaient originaires du Cameroun, 1 du Gabon. Les diagnostics pour thalassémies ont été pratiqués chez 4 Italiennes venant d'ltalie ou de Belgique, 1 Tunisienne, 1 Marocaine.

\section{II. - Diagnostic anténatal et isofocalisation.}

La méthode « classique » nécessite un équipement chromatographique coûteux et requiert un travail important. Les risques d'échecs techniques ne sont pas nuls. Notre équipe a récemment comparé les résultats obtenus grâce à cette méthode avec ceux fournis par l'isofocalisation des échantillons obtenus par fœtoscopie. La technique utilisée ne nécessite aucune incorporation d'acides aminés radioactifs, et utilise le matériel standard d'isofocalisation analytique vendu par LKB ou Pharmacia ; $10 \mu$ l d'hémolysat fołal débarrassé d'une éventuelle contamination par les érythrocyies maternels sont utilisés. Cette méthode distingue très aisément les fœtus $A / A, A / S, S / S, A / C$ et $S / C$ d'une pari en dépit de la présence à la $20^{\circ}$ semaine d'une très forte proportion d'Hb $F$ eł de 10 p. 100 d'Hb F acétylée au point isoélectrique proche de celui de l'Hb A. La figure 8 représente l'aspect obtenu avec 8 prélèvements à risque drépanocytaire et 7 prélèvements à risque thalassémique, 4 échantillons à risque drépanocytaire ne contenaient que de l'Hb A, 4 échantillons contenaient de la $A$ et de la $\mathrm{S}$. Dans tous les cas le diagnostic a concordé avec celui produit par la méthode classique (tabl. 2).

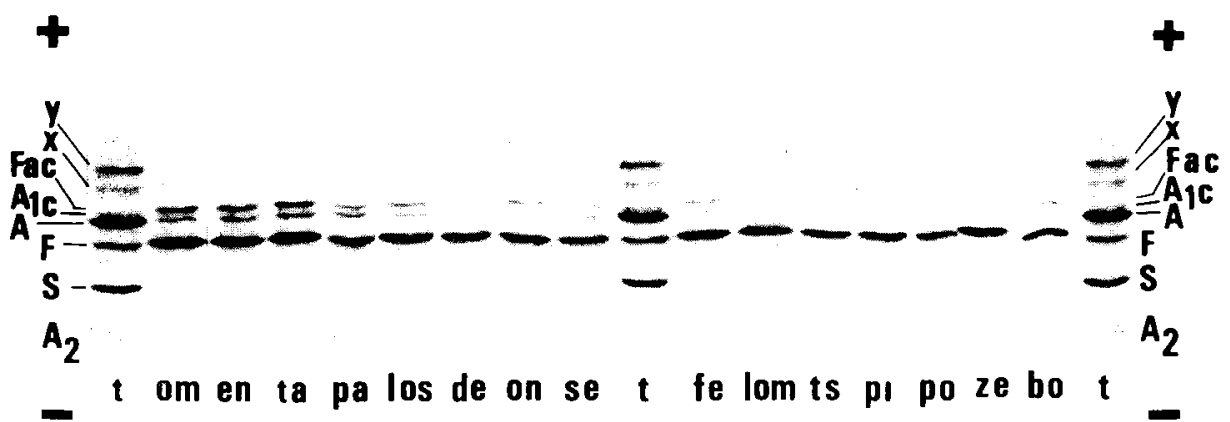

FIG. 8. - Isoélectrofocalisation de globules rouges de 15 fœtus à risque drépanocytaire OM., EN., TA., PA., LOS, DE., ON., SE. et $\beta$ thalassémique FE., LOM., TS., PI., PO., ZE., BO. ; T., est un mélange témoin comprenant $X: J$. Calabria, $Y: J$. Broussais.

Dans le cas des $\beta^{0}$ thal. le diagnostic est très aisé. De façon inattendue, le diagnostic fut possible également dans le cas de $\beta^{+}$thal. Les quantités d'Hb A présentes à la 20 e semaine dans les globules de $\beta^{+}$thal. semblent être très faibles et la différence semble être suffisante pour permettre de distinguer les homozygotes des hétérozygotes. Cette technique a été utilisée dans 17 diagnostics prénataux et contrôlée à l'aide de 7 fœetus homozygotes pour la $\beta^{0}$ thalassémie. Dans tous les cas les réponses entre l'isofocalisation et la technique classique ont été identiques. distance.

Cette technique, outre sa simplicité, permet une exploitation des prélèvements à 


\section{III. - Conséquences du diagnostic anténatal.}

Le diagnostic anténatal a pour but de permettre à certains couples encourant un risque élevé d'avoir des enfants anormaux, de donner naissance à des enfants normaux ou hétérozygotes si tel est leur désir. Les investigations anténatales peuvent montrer que le fœetus est atteint de la forme homozygote de l'hémoglobinose et ce diagnostic conduit à l'interruption de grossesse car toute idée de thérapeutique foetale est actuellement du domaine de la science-fiction. L'interruption de grossesse pose des problèmes éthiques et peut être l'objet d'un débat faisant intervenir des considérations morales et religieuses. Mais ces problèmes sont ceux du diagnostic anténatal en général et notre propos n'est pas d'en discuter ici. Précisons toutefois que le diagnostic anténatal, en ce qui concerne les hémoglobinopathies, conduit le plus souvent à la poursuite d'une grossesse dont le terme est la naissance d'un enfant sain (normal ou porteur du trait). Il est fréquent de voir les couples à haut risque ayant déjà un ou deux enfants malades demander systématiquement l'interruption de toute grossesse, ce qui est rendu plus simple par la loi récente de libéralisation de l'avortement. Ce diagnostic prénatal permet alors de sauver 3 fœu sur 4.

Les échecs sont possibles : 3 sur 15 dans une série récente d'Alter et al. (1), 2 sur 25 dans une série de Hobbins et Mahoney (12). Il s'agissait dans ces derniers cas d'un échec lié au prélèvement ; même après deux tentatives, les auteurs n'ont pu obtenir de cellules fœtales. Nous avons nous-mêmes subi 4 échecs sur 32 prélèvements. Ceux-ci semblent être inversement proportionnels avec la fréquence des prélèvements ; ils sont survenus dans la période initiale de nos tentatives.

Des erreurs ont été enregistrées au début de l'utilisation de ces nouvelles méthodes de diagnostic mais elles sont rares et les progrès techniques ont diminué notablement ce risque.

Le risque maternel est quasi inexistant si les prélèvements sont pratiqués en milieu très spécialisé, par des obstétriciens entraînés et bien équipés. L'interruption de grossesse après le $3^{e}$ mois comporte un certain risque mais il est certainement diminué par l'utilisation des prostaglandines.

Le danger de traumatisme fœetal par contre est à prendre en considération surtouł au cours des prélèvements aveugles par placentocentèse. II est dans ce cas notable (5 à 10 p. 100) et directement en rapport avec le nombre de ponctions. Il est moindre lorsque le prélèvement est fait sous le contrôle d'un fœtoscope : 1 mort fœtale pour 50 fœtoscopies pour Rodeck (21) aucune mort fœtale sur une série de 22 fœtoscopies chez des femmes ayant mené à terme leur grossesse pour Hobbins (12). Nous n'avons jusqu'ici, mais notre recul est très court, observé aucune anomalie de grossesse ou de lésion fœtale (un accouchement à 6 mois et demi chez une mère éclampsique ne semble pas pouvoir être relié au prélèvement de sang fœetal effectué chez cette femme).

\section{IV. - Diagnostic prénatal au niveau du gène.}

Le diagnostic prénatal «classique » des hémoglobinopathies s'appuie donc sur l'étude des chaînes d'hémoglobines, produits finaux des gènes. Or seules les cellules différenciées érythropoiétiques effectuant ces synthèses, une telle approche rendait impossible le diagnostic prénatal à l'aide de fibroblastes du liquide amniotique, maté- 
riel très utilisé pour le diagnostic anténatal des anomalies chromosomiques et de nombreuses enzymopathies : les fibroblastes en culture n'expriment pas le génome hémoglobine. Les progrès très rapides réalisés ces dernières années dans l'analyse directe du matériel génétique de l'Hb (isolement des messagers, fabrication de cDNA à partir de ceux-ci et à l'aide de réverse transcriptase, mise au point de méthodes de dosage génique à l'aide de ce matériel, par des techniques d'hybridation) ont permis de développer une nouvelle technologie utilisant les fibroblastes amniotiques. Kan a ainsi pu faire le diagnostic prénatal des $\alpha$ thalassémies en hybridant avec les sondes radioactives ad hoc (cDNA) du DNA fœtal extrait de cultures de fibroblastes amniotiques. Cette technique présente néanmoins le défaut d'être assez longue, d'autre part du DNA maternel contaminant peut éventuellement gêner les interprétations. Une nouvelle phase vient de s'ouvrir avec l'utilisation d'enzymes de restriction qui permettent une analyse par hybridation en milieu solide infiniment plus performante que l'hybridation en phase liquide utilisée jusqu'alors. Cette technologie a débouché dans 2 directions. Kan et Dozy (18) viennent de montrer que chez 80 p. 100 des sujets drépanocytaires un site de restriction pour l'enzyme $\mathrm{Hpa} I$ manquait, cependant qu'un nouveau site était révélé légèrement plus loin que le premier de l'extrémité $3^{\prime}$ du gène $\beta$. Les parents de ces sujets se présentent comme des hétérozygotes pour les sites en question possédant à la fois le site normal et le site « lié à l'état drépanocytaire 》. Le génome normal montre d'ailleurs une autre hétérogénéité. Ces caractéristiques sont objectivées par une électrophorèse à travers un tamis moléculaire suivie d'une hybridation in sifu à l'aide de cDNA radioactif. Les résultais obtenus sont représentés sur la figure 9 . Cetfe fechnique

FIG. 9. - Les blocs représentent les gènes $\beta$. Les flèches les sites de coupure par l'enzyme de restriction $\mathrm{Hpa} \mathbf{I}$; $\mathrm{kb}$ : kilobases ; $\mathrm{A}$ : gène $\beta^{A} ; S:$ gène $\beta^{S}$.

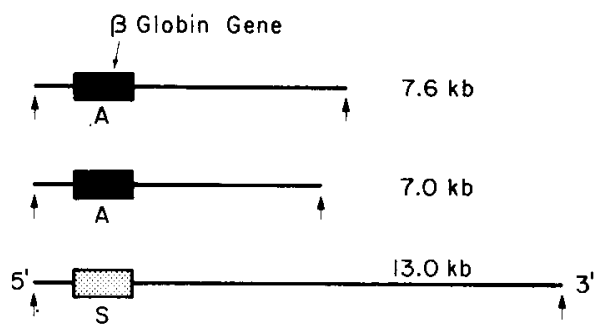

a permis à Kan et Dozy d'effectuer un diagnostic prénatal de drépanocytose à partir de fibroblastes contenus dans $15 \mathrm{ml}$ de liquide amniotique. Aucune culture n'est nécessaire. Le diagnostic est obtenu dans les 8 jours. Les avantages de cette technique sont éclatants. Si les premiers résultats sont confirmés, le diagnostic prénatal pourrait être étendu à des régions ne possédant pas une infrastructure en laboratoire très développé, mais des centres capables néanmoins de faire des amniocentèses. Les prélèvements envoyés congelés seraient traités dans des laboratoires spécialisés. D'autre part, même dans les régions bien équipées en laboratoires, cette technique serait utilisée eu égard aux avantages considérables que présente l'amniocentèse sur le prélèvemeni de sang. Une étape préalable fondamentale doit néanmoins être franchie : il faut savoir si le phénomène de liaison entre un site de restriction spécifique et le gène $\beta S$ est très largement répandu.

De toute manière même si les premiers résultats sont confirmés avec $80 \mathrm{p} .100 \mathrm{de}$ sujets transmetfeurs à la fois du gène $\beta S$ et du site $13 \mathrm{~KB}$, cela ne représente que 60 à 70 p. 100 de couples dont les 2 éléments sont transmetteurs du site 13 KB. Si l'un des 
parents ne le possède pas, le diagnostic ne peut être réalisé de la sorte. Ceci implique une éfude préalable à toute décision sur les populations susceptibles de bénéficier du diagnostic prénatal. Nos résultats préliminaires indiquent que la liaison gène $\beta S$ site $13 \mathrm{~KB}$ est d'une bonne fréquence à la Martinique et avoisine 80 p. 100 . L'étude est actuellement poursuivie. De toute façon, même si la fréquence est suffisante sur le plan statistique, cela ne dispensera absolument pas de faire un phénotypage systématique des 2 parents d'un couple à risque avant chaque diagnostic prénatal. Une réserve d'importance existe d'ailleurs toujours : celle de la grossesse illégitime. La méconnaissance du phénotype du père réel rend impossible l'interprétation des résultats obtenus sur le fœtus. Cet inconvénient n'existe évidemment pas avec la méthode classique où l'on phénotype directement les hémoglobines de l'enfant et non un gène lié à celui de la chaîne $\beta$ de globine.

II n'en demeure pas moins que sur le plan conceptuel, les résultats obtenus par Kan ont une portée considérable. Il est clair que la notion de sites de restriction modifiés liés à une affection génétique peut être extrapolée à toutes les maladies génétiques. II n'est même pas besoin de connaître le gène impliqué pour pouvoir éventuellement appliquer la méthode. Un site particulier existe peut-être lié à la lésion hémophile. Sa mise en évidence transformerait complètement le problème, ô combien délicat, du diagnostic prénatal au cours de cette affection.

Cette technique a déjà pu être appliquée à certaines variétés très rares de thalassémies : $\delta \beta$ thalassémies, maladie de Lepore (19). Elle ne s'applique à l'heure actuelle pas aux formes communes de $\beta$ thalassémies. Des recherches actives à l'aide d'un grand nombre d'enzymes de restriction sont menées dans l'espoir de trouver un site intra ou extra génique caractéristique de cette affection.

Le diagnostic prénatal des hémoglobinopathies offre un exemple tout à fait remarquable d'une bonne corrélation entre recherche fondamentale et recherche appliquée. Ce sont les progrès des ultramicrométhodes d'analyse des protéines qui ont permis entre autres le développement de la méthode « classique » de diagnostic prénatal. De même la connaissance des différences de composition enzymatique de l'érythrocyte au cours de l'ontogenèse a été à l'origine de la technique d'hémolyse différentielle des hématies maternelles contaminant les prélèvements folaux.

La méthode de Kan de diagnostic sur le DNA des fibroblastes a été une application pratique quasiment immédiate des techniques de cartographie du DNA développées à partir des travaux de Sutherland qui ne remontent qu'à 1975. A l'inverse, c'est d'une recherche appliquée de méthode nouvelle de diagnostic prénatal qu'est sortie la notion tout à fait fondamentale de la liaison entre un gène pathologique, le gène $\beta^{\text {s }}$ et une situation particulière vis-à-vis d'une enzyme de restriction. C'est en effet une notion tout à fait fondamentale que celle de l'existence d'une « différence génétique » liée à une mutation ponctuelle mais située en dehors du gène incriminé.

Il est évident qu'une recherche génétique, anthropologique, phylogénique va découler d'une observation provenant d'une recherche très appliquée. II n'est pas inutile de souligner que ce n'est que parce que des chercheurs fondamentalistes se sont intéressés à ce problème de recherche appliquée que ces observations ont pu être faites. 
Remerciements. - Nous remercions la Fondation pour la Recherche Médicale Française, la Délégation Générale de la Recherche Scientifique et Technique pour les décisions d'aide $n^{\circ}$ 79-7-0485 et no 78-7-0345 ainsi que l'Institut National de la Santé et de la Recherche Médicale, ATP no 56-78-88 et no 36-76-68.

\section{Références}

1. ALTER B. P., MODELL C. B., FAIRWEATHER D., 1976. Prenatal diagnosis of hemoglobinopathies. A review of 15 cases. New Engl. J. Med., 295, 1437-1443.

2. ALTER B. P., METZGER J., YOCK P. G., ROTHCHILD S. B., DOVER G. J., 1979. Selective hemolysis of adult red blood cells : an aid to prenatal diagnosis of hemoglobinopathies. Blood, 53, 279-287.

3. BASSET P., BEUZARD Y., GAREL M. C., ROSA J., 1978. Isoelectric focusing of human hemoglobin : its application to screening, to the characterization of 70 variants and to the study of modified fractions of normal hemoglobins. Blood, 51, 971-982.

4. BOYER S. H., NOYES A. N., BOYER M. C., 1976. Enrichment of erythrocytes of fetal origin from adult -fetal blood mixtures via selective hemolysis of adult blood cells : an aid to antenatal diagnosis of hemoglobinopathies. Blood, 47, 883-897.

5. CHANG H., MODELL C. B., ALTER B. P., 1975. Expression of the $\beta$-thalassemia gene in the first trimester fetus. Proc. nat. Acad. Sci. USA, 72, 3633-3637.

6. CIVIDALLI G., NATHAN D. G., KAN Y. W., 1974. Relation of beta to gamma synthesis during the first trimester. An approach to prenatal diagnosis of thalassemia. Pediat. Res., 8, 553-560.

7. DUBART A., GOOSSENS M., BEUZARD Y., MONTPLAISIR N., TESTA U., BASSET P., ROSA J., 1979. Prenatal diagnosis of hemoglobinopathies by isoelectric focusing (soumis à Blood).

8. GOOSSENS M., BEUZARD Y., ROSA J., 1976. Problèmes biochimiques posés par le diagnostic anténatal des hémoglobinopathies. Coll. INSERM A. BOUÉ. Diagnostic prénatal, 61, 263-273.

9. GOOSSENS M., BEUZARD Y., HENRION R., ROSA J., 1978. Diagnostic anténatal des hémoglobinopathies. Arch. franç. Péd., 35, 660-669.

10. HOBBINS J., MAHONEY M., 1975. Fetal blood drawing. Lancet, 11, 107-109.

11. HOBBINS J., MAHONEY M., 1974. In utero diagnosis of hemoglobinopathies. Technic for obtaining fetal blood. New. Engl. J. Med., 290, 1065-1067.

12. HOBBINS J. C., MAHONEY M. J., 1977. Fetoscopy in continuing pregnancies. Am. J. Obstet Gynecol., 129, 440-442.

13. KAN Y. W., VALENTI C., GUIDOTTI R., 1974. Fetal blood sampling in utero. Lancet, 1, 79-80.

14. KAN Y. W., GOLBUS M. S., DOZY A. M., 1976. Prenatal diagnosis of $\alpha$ thalassemia : clinical application of molecular hybridization. New Engl. J. Med., 295, 1165-1167.

15. KAN Y. W., GOLBUS M. S., TRECARTIN E. F., 1977. Prenatal diagnosis of $\beta$-thalassemia and sickle cell anaemia. Experience with 24 cases. Lancet, 1, 269-271.

16. KAN Y. W., DOZY A. M., ALTER B. P., 1972. Detection of the sickle gene in the human fetus. New. Engl. J. Med., 287, 1-5.

17. KAN Y. W., NATHAN D. G., CIVIDALLI G., 1974. Concentration of fetal red blood cells from a mixture of maternal and fetal blood by anti-i serum. An aid to prenatal diagnosis of hemoglobinopathies. Blood, 43, 411-415.

18. KAN Y. W., DOZY A., 1978. Antenatal diagnosis of sickle cell anemia by DNA analysis of amniotic fluid cells. Lancet, 11, 910-911.

19. ORKIN S. H., ALTER B. P., ALTAY C., MAHONEY M. J., LAZARUS H. HOBBINS J. C., NATHAN D. G., 1978. Endonuclease mapping to detect globin gene deletion in the prenatal diagnosis of thalassemia. New Engl. J. Med., 299, 166-172.

20. ORSINI A., 1977. Aspects médico-sociaux des hémoglobinoses. Arch. franç. Pèd., 34, 393-398.

21. RODECK C. H., CAMPBELL S., 1978. Sampling of pure fetal blood by fetoscopy in second trimester of pregnancy. Brit. Med. J., 2, 728-730.

22. VALENTI C., 1973. Antenatal detection of hemoglobinopathies. A preliminary report. Am. J. Obstet. Gynecol., 115, 851-853. 